\title{
Optimized Amplitude-Dividing Beam Splitter Gratings for 4D STEM Holography
}

Andrew Ducharme ${ }^{1}$, Cameron Johnson ${ }^{2}$, Peter Ercius ${ }^{3}$ and Benjamin McMorran ${ }^{4}$

${ }^{1}$ University of Oregon, United States, ${ }^{2}$ University of Oregon, Eugene, Oregon, United States, ${ }^{3}$ Lawrence Berkeley National Laboratory, United States, ${ }^{4}$ United States

Material diffractive electron optics have the capacity to sculpt the electron matter wave arbitrarily up to nanofabrication resolution limits. The resulting hologram can impart both phase and amplitude modulation, combining significant design flexibility [1-2] with the sub-nanoscale imaging of the TEM to support a multitude of applications [3-6]. This level of control, however, requires maximizing grating output into the proper diffraction order to ensure a detectable proportion of electrons interact with the sample. Simultaneously, all other order modes must be minimized to prevent adding destructive levels of noise to the measurement.

One specific application of beam shaping is the use of straight diffraction gratings to create spatially separated, well-defined diffraction in the transverse plane. This can lead towards interferometric nanoscale phase imaging [7-8], probing of coherence properties of free electrons [9], and grating based MachZehnder interferometry inside a TEM [10-11], which itself can enable dose sensitive interaction-free measurements [12].

Here, we use XeF2 gas-assisted ion beam milling of SiN films to create uniform $300 \mathrm{~nm}$ pitch diffraction gratings. Previous gratings were designed for $300 \mathrm{keV}$ operation, but we manufactured new gratings optimized for $80 \mathrm{keV}$ operation in order to image materials below the knock-on damage threshold of the sample. This recent run demonstrated an average diffraction efficiency $\eta$, depicted in Fig 1(a), of 11.6\% and $34.1 \%$ in the zeroth and first diffraction orders respectively. The optimal individual grating had diffraction efficiencies for the same orders of $7.5 \%$ and $34.4 \%$. Fig 1(b-d) shows the diffraction efficiency of this grating, its TEM diffraction pattern, and the output intensity.

The beam splitter will be inserted into the TEAM 0.5 instrument which is equipped with the 4D Camera for $87,000 \mathrm{~Hz}$ 4D-STEM imaging. This will allow low-dose and rapid imaging with our gratings over a large field of view making this a generalizable technique for nanoscale phase imaging, and could also be used for tomography or in situ experiments.

\section{References}

[1] Johnson, C.W. et al. Exact design of complex amplitude holograms for producing arbitrary scalar fields. Opt. Express 28, 17334-17346 (2020).

[2] Johnson, C.W., Bauer D.H., and McMorran B.J. Improved control of electron computer-generated holographic grating groove profiles using ion beam gas-assisted etching. Appl. Opt. 59, 1594-1601 (2020). [3] Ophus, C., Ciston, J., Pierce, J. et al. Efficient linear phase contrast in scanning transmission electron microscopy with matched illumination and detector interferometry. Nat. Commun. 7, 10719 (2016). 


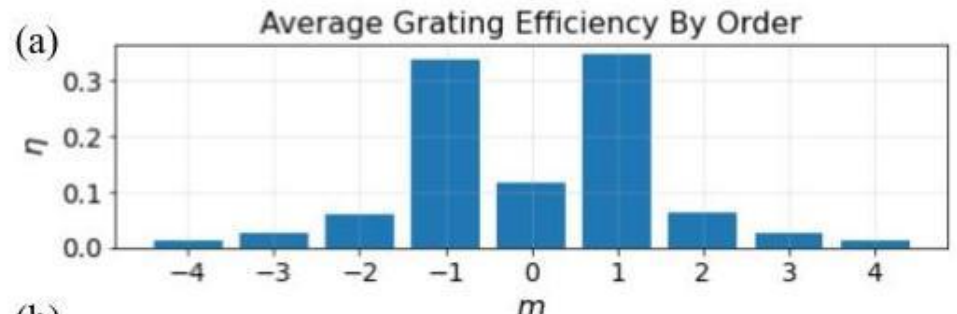

(b)
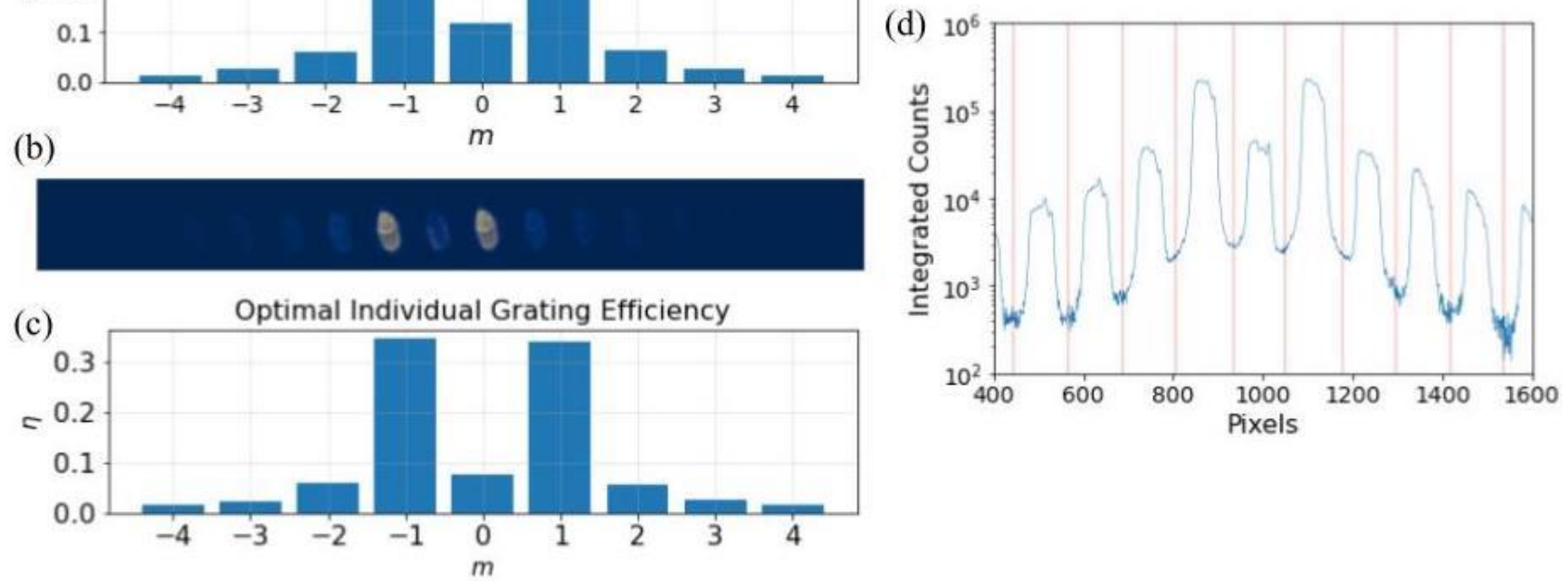

Figure 1. Diffraction grating performance. (a) Average measured diffraction efficiency $\eta$ in the zeroth through \pm 4 orders $m$. (b) Diffraction pattern for optimal individual grating. Image is defocused to show aperture image. (c) Maximum measured diffraction efficiency for an individual grating. (d) Grating output intensity for optimal individual grating. Red lines mark the minima used to demarcate between orders.

[4] Negi, D.S., Idrobo, J.C. and Rusz, J. Probing the localization of magnetic dichroism by atomic-size astigmatic and vortex electron beams. Sci. Rep. 8, 4019 (2018).

[5] Guzzinati, G. et al. Electron Bessel beam diffraction for precise and accurate nanoscale strain mapping. Appl. Phys. Lett. 114, 243501 (2019).

[6] Linck, M. et al. Aberration corrected STEM by means of diffraction gratings. Ultramicroscopy 182, 36-43 (2017).

[7] Harvey, T.R. et al. Interpretable and Efficient Interferometric Contrast in Scanning Transmission Electron Microscopy with a Diffraction-Grating Beam Splitter. Phys. Rev. Appl. 10, 061001 (2018).

[8] Yasin, F.S. et al. Probing Light Atoms at Subnanometer Resolution: Realization of Scanning Transmission Electron Microscope Holography. Nano Lett. 18, 7118-7123 (2018).

[9] Poptapov, P.L. et al. Experiments on inelastic electron holography. Ultramicroscopy, 106, 1012-1018 (2006).

[10] Gronniger, G., Barwick, B., and Batelaan, H. A three-grating electron interferometer. New J. Phys. 8, 224 (2006).

[11] Agarwal, A. et al. A nanofabricated, monolithic, path-separated electron interferometer. Sci. Rep. 7, 1677 (2017).

[12] Turner, A. et al. Single Electron Interferometry: A Step Toward Quantum Electron Microscopy. Microscopy and Microanalysis 25, 1712-1713 (2019).

[13] This work was supported by the National Science Foundation under Grant No. 2012191. 